\title{
Therapy with transcutaneous administration of imiquimod combined with oral administration of sorafenib suppresses renal cell carcinoma growing in an orthotopic mouse model
}

\author{
TAKASHI KARASHIMA ${ }^{1}$, KEIKO UDAKA ${ }^{2}$, MAYUMI NIIMURA ${ }^{1}$, KATSUHIDE SUZUKI $^{2}$, \\ HIROTO OSAKABE ${ }^{1}$, TSUTOMU SHIMAMOTO ${ }^{1}$, SATOSHI FUKATA ${ }^{1}$, KEIJI INOUE ${ }^{1}$, \\ NAOTO KURODA ${ }^{3}$, MOTOHARU SEIKI ${ }^{4}$ and TARO SHUIN ${ }^{1}$ \\ Departments of ${ }^{1}$ Urology and ${ }^{2}$ Immunology, Kōchi Medical School, Nankoku, Kōchi 783-8505; \\ ${ }^{3}$ Department of Diagnostic Pathology, Kōchi Red Cross Hospital, Kōchi, Kōchi 780-0062; \\ ${ }^{4}$ Medical Innovation Center, Kōchi Medical School, Nankoku, Kōchi 783-8505, Japan
}

Received December 24, 2015; Accepted February 23, 2017

DOI: $10.3892 / 01.2017 .6235$

\begin{abstract}
Imiquimod is an imidazoquinoline immune response modifier that is used in antiviral and antiallergic creams. Combination therapy using transcutaneous imiquimod and oral sorafenib was previously demonstrated to reduce the tumor burden of renal cell carcinoma growing cutaneously in a mouse model. In the present study, an orthotopic mouse model was used to investigate whether combined treatment with oral sorafenib and transcutaneous imiquimod inhibited renal cell carcinoma growing in the kidney. Kidneys of female BALB/c mice were orthotopically implanted with RENCA mouse kidney cancer cells, and the mice were transcutaneously treated with cream containing imiquimod and/or with orally administered sorafenib 5 days following cell implantation. Tumor burden and incidence were determined 28 days following the start of therapy. Splenocyte activity was quantified using the ${ }^{51} \mathrm{Cr}$ release assay and the fluorescence-activated cell sorting assay with cluster of differentiation (CD) 4 and CD8 antibodies. Imiquimod, sorafenib and combination therapy were tolerated well. A combination of transcutaneous imiquimod and oral sorafenib inhibited the growth of RENCA tumors in the kidney significantly compared with the control. The ${ }^{51} \mathrm{Cr}$ release
\end{abstract}

Correspondence to: Dr Takashi Karashima, Department of Urology, Kochi Medical School, Kohasu Oko, Nankoku, Kōchi 783-8505, Japan E-mail: karasima@kochi-u.ac.jp

Abbreviations: RCC, renal cell carcinoma; IFN- $\alpha$, interferon alpha; IQM, imiquimod; TKI, tyrosine kinase inhibitor; VEGFR, vascular endothelial growth factor receptor; flt3, Fms-like tyrosine kinase 3; PDGFR, platelet-derived growth factor receptor; CD, cluster of differentiation; FACS, fluorescence-activated cell sorting; CTL, cytotoxic T lymphocyte

Key words: renal cell carcinoma, imiquimod, sorafenib, cytotoxic $\mathrm{T}$ lymphocyte, transcutaneous administration assay demonstrated that transcutaneous imiquimod therapy significantly induced the release of ${ }^{51} \mathrm{Cr}$ from RENCA cells compared with the control. The fluorescence-activated cell sorting assay demonstrated that transcutaneous imiquimod therapy induced $\mathrm{CD}^{+}$and $\mathrm{CD}^{-}{ }^{-}$splenocytes compared with the control. In summary, the results of the present study demonstrated that combined treatment with transcutaneous imiquimod and oral sorafenib may be a promising strategy for the treatment of patients with renal cell carcinoma.

\section{Introduction}

Renal cell carcinoma (RCC) is the most common malignancy that arises from the kidney, and is known to respond to immunotherapy. Non-specific immunotherapy, including interferon $\alpha$ (IFN- $\alpha)$, interleukin-2 and immune checkpoint agents including programmed cell death-1 (1), programmed cell death-ligand 1 (2) and cytotoxic T-lymphocyte-associated protein-4 (3) antibodies may promote survival and be tolerated well in patients with RCC.

Imiquimod [3-(2-methylpropyl)-3,5,8-triazatricyclo (7.4.0.02,6) trideca-1 (9), 2 (6), 4,7,10,12-hexaen-7-amine; IQM], an imidazoquinoline compound, is a Toll-like receptor-7 ligand that induces production of cytokines, including IFN- $\alpha$, in a variety of cell types (4). IQM is often used as an antiviral (4) and antiallergic (5) agent in a cream form. The anti-tumor effects of IQM have previously been demonstrated in patients with skin neoplasia, including actinic keratosis (6), basal cell carcinoma (7) and melanoma in situ (8).

$\mathrm{RCC}$ is primarily hyper-angiogenic and metastasizes by hematogenous spread to visceral organs. Therefore, anti-angiogenic therapies are a rational strategy against advanced RCC. Anti-angiogenic tyrosine kinase inhibitors (TKIs) are widely used to treat patients with cancer, including RCC. Sorafenib was the first TKI used to treat RCC, developed by Bayer AG (Leverkusen, Germany) and Onyx Pharmaceuticals, Inc. (San Francisco, CA, USA). Sorafenib was designed as a multi-targeted small molecule inhibitor of Ser/Thr kinases and several receptor tyrosine kinases including vascular 
endothelial growth factor receptor (VEGFR)-2, VEGFR-3, Fms-like tyrosine kinase 3 (flt3), platelet-derived growth factor receptor (PDGFR)-B, and c-kit (9).

The combination of transcutaneous IQM administration and oral sorafenib treatment was previously demonstrated to reduce the tumor burden of cutaneous RCC, and was well tolerated in a mouse model (10). The aim of the present study was to determine whether transcutaneous IQM reduced RCC in a visceral organ and whether combined transcutaneous IQM and oral sorafenib treatment was effective as a novel therapeutic strategy to reduce orthotopic RCC in the kidney. The results of the present study may help to develop a combination therapy with activated natural immunity for patients with advanced RCC, for example IQM and TKI.

\section{Materials and methods}

Cell lines and culture conditions. The mouse RCC cell line RENCA was obtained from the American Type Culture Collection (Manassas, VA, USA). RENCA was established from a tumor that arose spontaneously as a renal cortical adenocarcinoma in BALB/c mice. The cells were maintained as monolayer cultures in RPMI-1640 medium (Wako Pure Chemical Industries, Ltd., Osaka, Japan) supplemented with 10\% fetal bovine serum (FBS; Thermo Fisher Scientific K.K, Yokohama, Japan). Adherent monolayer cultures were maintained on plastic and incubated at $37^{\circ} \mathrm{C}$ under a mixture of 5\% $\mathrm{CO}_{2}$ and $95 \%$ air. The cultures were maintained for no longer than 12 weeks following recovery from frozen stocks.

Reagents. IQM (Beselna), which is often used in cream form as an antiviral agent, was obtained from Mochida Pharmaceutical Co., Ltd. (Tokyo, Japan) $(4,11)$. Sorafenib (Nexavar) was provided by Bayer AG (Leverkusen, Germany) (9).

Animals. A total of 20 female 6-week-old BALB/c Cr Slc mice and athymic BALB/c A Jc1-nu nude mice (body weights, 15-20 g) were obtained from Japan SLC, Inc. (Shizuoka, Japan). The mice were housed and maintained in specific pathogen-free conditions maintained at $20-26^{\circ} \mathrm{C}$, with access to feed ad libitum and a 12/12 light/dark cycle. The care and use of animals in the present study was approved by Kochi University Animal Care and Use Committee and conformed to international guidelines on the ethical use of animals. All efforts were made to minimize the number of experimental animals and their suffering.

Implantation of tumor cells. To produce kidney tumors, RENCA cells were harvested from subconfluent cultures by brief exposure to $0.25 \%$ trypsin and $0.02 \%$ EDTA. Trypsinization was stopped with RPMI-1640 medium containing 10\% FBS, and the cells were washed once in serum-free RPMI-1640 medium and resuspended in RPMI-1640 medium. Nude mice were anesthetized with $10 \mathrm{~g} / \mathrm{kg}$ Nembutal intraperitoneally (i.p.; Abbott Laboratories, Lake Bluff, IL, USA). A flank incision was made, and tumor cells were injected into the renal subcapsule of the kidney upper pole. The formation of a bulla indicated a satisfactory injection. To prevent leakage of cells to the surrounding tissue, a cotton swab was held for 1 min over the site of injection. The kidney was returned to the abdominal cavity and the abdominal wall closed with a single layer of metal clips (12). The animals tolerated the surgical procedure well and no anesthesia-associated mortality occurred.

Treatment. Mice were randomized into one of four groups ( $\mathrm{n}=10$ for each) 5 days following tumor cell implantation to receive: Daily oral administration of carboxymethylcellulose-based vehicle solution, daily cutaneous administration of $40 \mathrm{mg} / \mathrm{kg}$ IQM as 5\% Beselna cream, daily oral administrations of $10 \mathrm{mg} / \mathrm{kg}$ sorafenib or combination therapy with IQM and sorafenib. The mice were treated for 28 days, following which the tumor weight $(\mathrm{g})$ was determined. All mice were prepared by shaving the hair on the back using electric hair clippers, followed by an application of depilatory cream to remove fine hair. The depilatory cream was applied for $1 \mathrm{~min}$ to the skin, followed by rinsing with warm water. The hair removal was performed every 3 days (10).

Immunization and treatment of mice. For immunization and treatment, $1 \times 10^{7}$ RENCA cells, irradiated with $50 \mathrm{~Gy}$, were i.p. injected into $\mathrm{BALB} / \mathrm{c}$ mice, once a week, five times. A daily transcutaneous treatment of IQM $(40 \mathrm{mg} / \mathrm{kg})$ was applied to the back skin.

Quantification of cytotoxicity of splenocytes by ${ }^{5 l} \mathrm{Cr}$ release assay. To measure the cytotoxic activity of splenocytes, the ${ }^{51} \mathrm{Cr}$ release assay was performed as described previously (13). Briefly, a week following the last immunization of mice by i.p. injection with irradiated RENCA cells, the mice were sacrificed and $1 \times 10^{6}$ splenocytes were harvested and cultured with $1 \times 10^{5}$ irradiated RENCA cells as stimulators in 6-well plates. Five days later, splenocytes of various effector/tumor cell (E/T) ratios were plated with $1 \times 10^{4}$ RENCA cells labeled with ${ }^{51} \mathrm{Cr}$ isotope, in a final volume of $200 \mu \mathrm{l}$, in 96-well U-bottom plates. Following $5 \mathrm{~h}, 130 \mu \mathrm{l}$ supernatant was transferred into $1.2 \mathrm{ml}$ micro-filtration tubes and radioactivity was determined by $\gamma$-counter (2470WIZARD2; PerkinElmer, Inc., Waltham, MA, USA). Percent specific lysis was calculated as follows: (Experimental release-spontaneous release) / (maximal release-spontaneous release) x100. Spontaneous and maximal releases were determined in the presence of RPMI-1640 medium supplemented with $10 \%$ FBS, or $0.1 \%$ Triton X-100, respectively.

Quantification of splenocytes expressing cluster of differentiation $(C D) 4$ and $C D 8$. Flow cytometric analysis was performed as described previously (14). Briefly, 5 days following preparation of the primary culture as described above, the cytotoxicity of splenocytes from mice treated with the control vehicle Cremophor EL (Sigma-Aldrich; Merck KGaA, Darmstadt, Germany) diluted to $25 \%$ with distilled water and IQM was analyzed by fluorescence-activated cell sorting (FACS). Splenocytes $\left(2 \times 10^{5}\right)$ were incubated with R-phycoerythrin-conjugated anti-mouse CD4 monoclonal antibody (dilution, 1:200; clone, RM4-5; cat. no. 50-0042, Tonbo Biosciences, San Diego, CA, USA), fluorescein isothiocyanate-conjugated anti-mouse CD8 monoclonal antibody (dilution, 1:100; clone, Ly2; Cedarlane Laboratories, Burlington, Ontario, Canada), and 7-amino-actinomycin D (BD Biosciences, Tokyo, Japan) for $20 \mathrm{~min}$ on ice. The 
antibodies had been diluted with stain buffer (BD Biosciences) Following washing with staining buffer (2\% FBS, $0.05 \% \mathrm{NaN}_{3}$ in phosphate-buffered saline), the splenocytes were resuspended with staining buffer, then flow cytometry was performed using a FACSCalibur flow cytometer and CellQuest software (FACStation ver 1.1; BD Biosciences, Franklin Lakes, NJ, USA).

Statistical analysis. The tumor volume was statistically analyzed using Prism software (Graph Pad Software V4.0) to perform Mann-Whitney $\mathrm{U}$ tests. $\mathrm{P}<0.05$ was considered to indicate a statistically significant difference.

\section{Results}

Combination of transcutaneous IQM and oral sorafenib as a treatment for BALB/c mice with orthotopic RENCA tumors. The present study aimed to determine whether a combination of transcutaneous IQM and oral sorafenib would inhibit RENCA cell growth when implanted into the kidneys of BALB/c mice. Therapy continued for 28 days and commenced 5 days following tumor implantation. The mice were monitored for progressive disease and, if they became moribund, were sacrificed. A combination treatment of IQM and sorafenib significantly decreased the weight of kidney tumors compared with tumors treated with the control vehicle $(\mathrm{P}<0.05$; Table I). Representative photographs of left kidneys bearing RENCA tumors that had been treated with control vehicle, IQM or sorafenib alone or IQM and sorafenib combined, are presented in Fig. 1.

Therapy with IQM increased the cytolysis of splenocytes against RENCA cells. To determine whether transcutaneous therapy with IQM increased the cytolytic activity of splenocytes against RENCA cells, RENCA cells irradiated with 60 Gy were injected into the peritoneal cavity of mice, once a week for a total of four times. Pairs of mice were treated with daily transcutaneous IQM or control vehicle for 4 weeks, then sacrificed and the spleens were removed. The cytotoxicity of the splenocytes was measured using the ${ }^{51} \mathrm{Cr}$ release assay (Fig. 2). The level of released ${ }^{51} \mathrm{Cr}$ identified cytotoxic activity as $\%$ specific lysis, and was significantly higher in the mice treated with IQM compared with those that received control vehicle. The \% specific lysis of splenocytes from each mouse treated with IQM was dependent on the E/T ratio.

IQM therapy induced CD8-positive and CD4-negative splenocytes. Splenocytes from mice treated with transcutaneous IQM were analysed, because IQM therapy was demonstrated to have a tumor regulatory activity in the data from the present study. The induction of splenocytes was assessed by FACS with CD4 and CD8 antibodies. The number of CD8-positive and CD4-negative splenocytes from mice treated with IQM (upper left of right two panels; 13.41 and 9.40; Fig. 3) was higher than that from the mice treated with control vehicle (upper left of left two panels; 5.45 and 2.81; Fig. 3).

\section{Discussion}

IQM is a prescription cream containing an imidazoquinoline, a class of compounds first synthesized in 1980, as an active
Table I. Tumorigenicity of mouse renal cell carcinoma growing in the kidney of BALB/c mice.

\begin{tabular}{lccc}
\hline & & \multicolumn{2}{c}{ Tumor weight $(\mathrm{g})$} \\
\cline { 3 - 4 } Therapy & Incidence & Median & Range \\
\hline Control & $10 / 10$ & 1.38 & $0.63-3.95$ \\
IQM & $9 / 9$ & 0.94 & $0.41-1.89$ \\
Sorafenib & $9 / 9$ & 1.23 & $0.48-1.72$ \\
Combination & $10 / 10$ & $0.48^{\mathrm{a}}$ & $0.19-1.69$
\end{tabular}

${ }^{\mathrm{a}} \mathrm{P}<0.05$ vs. control kidney tumor, as determined by the Mann-Whitney U test. IQM, imiquimod.

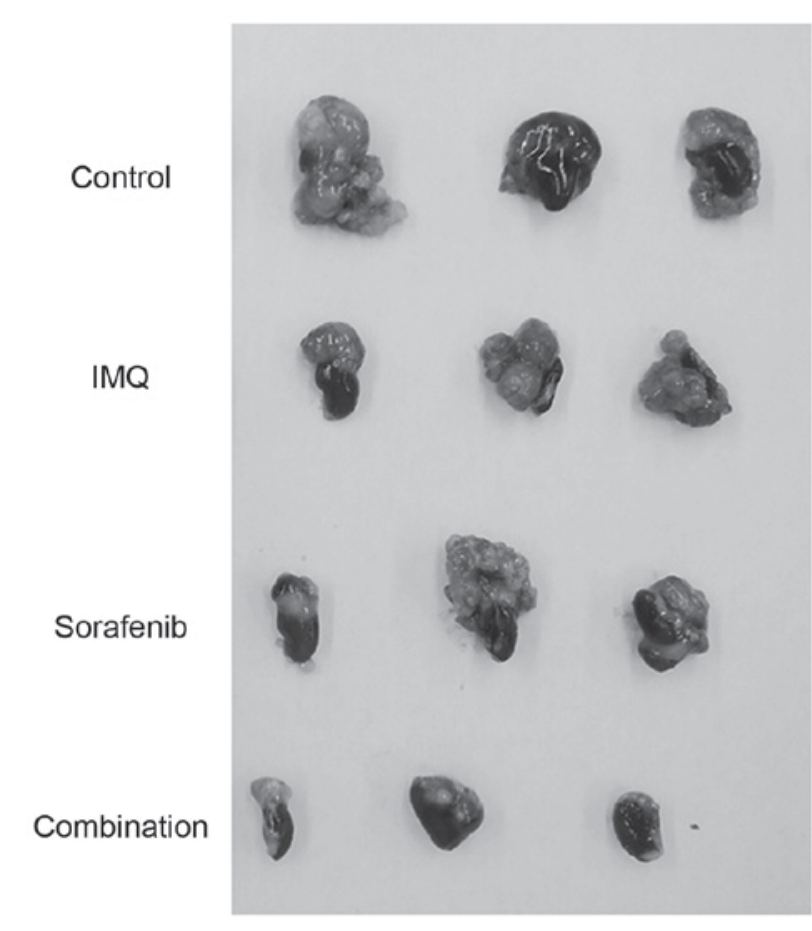

Figure 1. Renal cell carcinoma tumor growth in the kidney of BALB/c mice. The photographs are representative of kidneys bearing RENCA tumors that were treated with control vehicle (Cremophor EL diluted to 25\% with distilled water), cutaneous IQM, oral sorafenib or IQM and sorafenib combined. Therapy with IQM and/or sorafenib inhibited tumor growth in the kidney compared with the control vehicle. IQM, imiquimod.

ingredient. It is marketed by Meda AB (Solna, Sweden), Graceway Pharmaceuticals; Medicis Pharmaceutical Corporation (Scottsdale, AZ, USA) and iNova Pharmaceuticals (Sydney, Australia) under the trade names Aldara and Zyclara, and by Mochida Pharmaceutical Co., Ltd. as Beselna. Imidazoquinoline is an organic, double-cyclic molecule, and its compounds and derivatives act as cellular immune response modifiers. Several anti-tumor effects of IQM therapy have been reported, including immunomodulation (4), anti-angiogenesis (15), and the induction of tumor apoptosis by activating caspase-3 and reducing anti-apoptotic factors, including B cell lymphoma-2 $(16,17)$.

Sorafenib is a TKI developed for patients with metastatic, unresectable RCC and RCC that is resistant to typical 


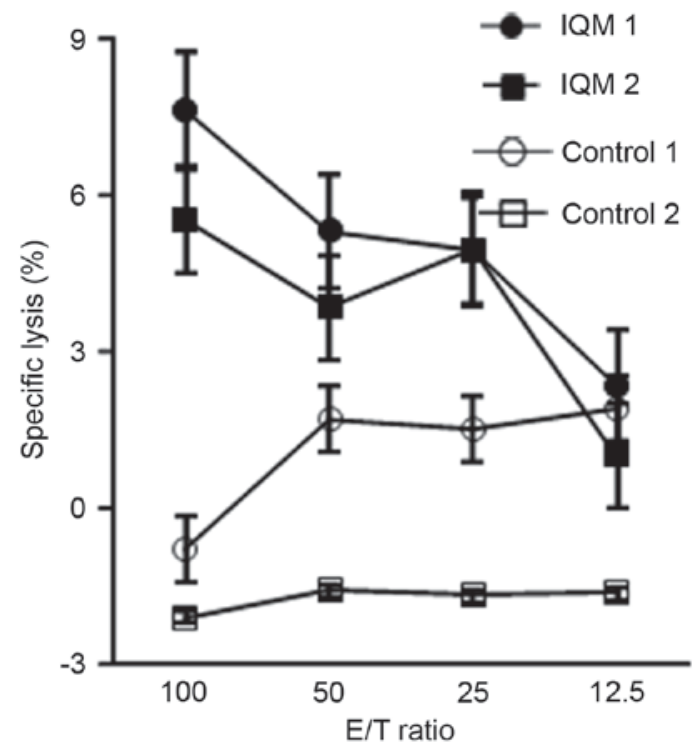

Figure 2. Cytolytic activity of splenocytes, determined by ${ }^{51} \mathrm{Cr}$ release assay. Daily cutaneous therapy with IQM enhanced the cytolytic activity of splenocytes against RENCA cells. Percentage specific lysis of each mouse (mouse 1 and 2) treated with IQM was dependent on the E/T ratio. IQM, imiquimod; $\mathrm{E} / \mathrm{T}$, effector/tumor cell.

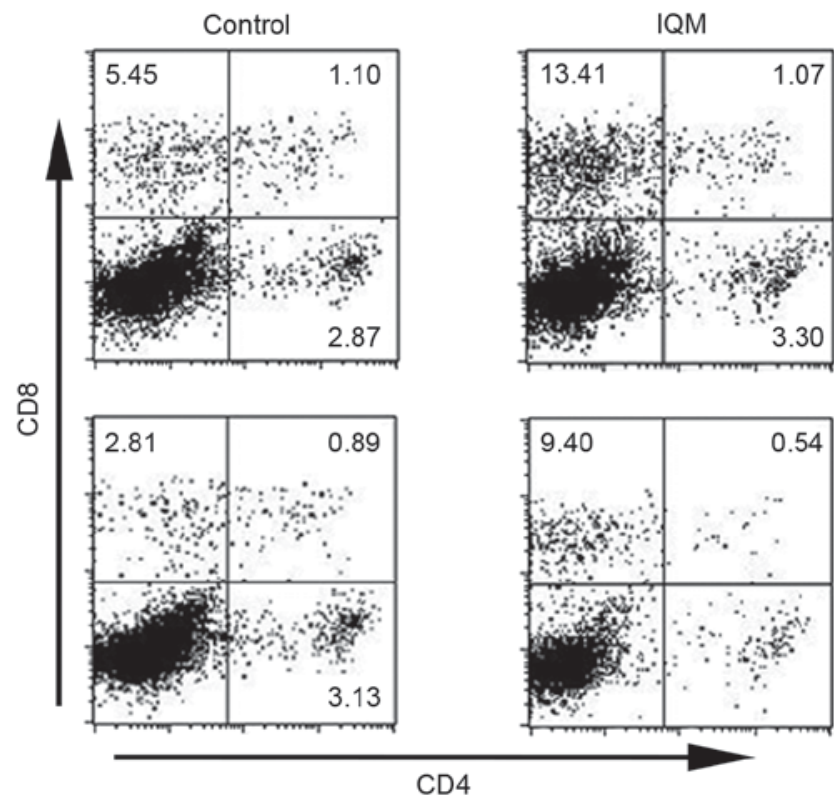

Figure 3. Population of CD4- and/or CD8-positive splenocytes from BALB/c mice treated with IQM, as determined by fluorescence-activated cell sorting. $\mathrm{BALB} / \mathrm{c}$ mice were injected intraperitoneally with irradiated RENCA cells, once a week, 4 times. Daily transcutaneous administration of IQM and control vehicle was commenced the day following injection. The splenocytes from each mouse were cultured with irradiated RENCA cells for 5 days, then analyzed by flow cytometry following staining with CD4 and CD8 antibodies on day 6. IQM therapy increased the number of tumor-specific $\mathrm{CD} 8^{+}$and CD4 T cells (upper left panel) compared with the control group (IQM, 13.41 and 9.40; control, 5.45 and 2.81). CD, cluster of differentiation; IQM, imiquimod.

chemotherapy and cytokine therapy (18). Immunomodulation by TKIs, including sorafenib, has previously been reported, which may have potential to modulate natural and adaptive immunity, and also to block the function of regulatory T cells while activating effector T cells in cancer $(19,20)$.
Transcutaneous administration of IQM was previously reported to significantly inhibit the growth of subcutaneous tumors in T-cell-competent mice, but not athymic nude mice (10); it was not clear whether the anti-tumor effect was produced by cytotoxic T lymphocytes (CTLs), because IQM may directly suppress the subcutaneous RENCA tumor. In the present study, a combination of transcutaneous IQM and oral sorafenib suppressed RCC growing in the kidney of orthotopic mouse models. Splenocytes demonstrated increased cytolysis in an E/T-ratio-dependent manner (Fig. 2), and the population of splenocytes contained CD8 positive and CD4 negative cells, indicating that CTLs were present (Fig. 3). These data demonstrated that the anti-tumor effect of transcutaneous IQM therapy proceeded via remote induction of tumor-specific CTLs, an effect that is synergistically activated by sorafenib therapy. Thus, the combination of transcutaneous IQM as a CTL inducer and oral sorafenib as a TKI represents a promising novel therapeutic strategy for patients with RCC.

There has been interest in non-invasive drug delivery and targeting for malignant neoplasms: Trans-orally, mucosally, nasally, vaginally, pulmonary, ophthalmically and cutaneously. In the past 30 years, transdermal systemic delivery of drugs has been explored. This would provide an attractive non-invasive alternative to medicines that require frequent injections, or to orally administered medicines that may elicit severe adverse events. Advantages of the transcutaneous route to systemically deliver drugs are convenient and painless administration, avoidance of gastrointestinal irritation, avoidance of significant first-pass metabolism due to degradation in the gastrointestinal tract or by the liver, reduced inter- and intra-patient variability, the tendency to confer a slow, sustained pharmacokinetic profile that is best suited to medicines that require prolonged exposures, leading to reduced frequency of dosing, improved patient compliance and acceptability of the drug therapy. Even in unconscious or nauseated patients, it is possible to terminate drug input simply by washing or peeling $(21,22)$. A wide range of transcutaneous products have been approved, providing acute and chronic treatment modalities for menopause and postmenopausal osteoporosis, contraception, angina, pain, smoking cessation, motion sickness, dementia, Alzheimer's disease, Parkinson's disease, attention deficit disorder and asthma $(23,24)$. These attest to the success of delivery via the skin and have been very effective adjuncts to oral therapies.

There are three principal disadvantages of transcutaneous drug delivery systems. The first is associated with the skin's barrier function, which severely constrains the amount of a drug that it is possible to absorb from a reasonable area of skin during a dosing period. The stratum corneum is a 10-20 $\mu \mathrm{m}$ thick barrier that is composed of corneocytes filled with keratin bundles, lipids and intercellular lipid lamellae packed tightly together. Even if the drug is sufficiently potent, its molecular weight should be less than $350 \mathrm{Da}$. Second, the pharmacokinetic and pharmacodynamic characteristics of the drug must be such that the relatively sustained and slow input provided by transdermal delivery makes sense. Drugs that it is possible to give orally once a day with reproducible bioavailability, or that are are well tolerated by the patient, do not need transcutaneous administration. Third, dermatitis is the most common dosing site adverse effect. The drug must not be locally irritating or sensitizing $(21,22,25)$. 
IQM has a molecular weight of $240.31 \mathrm{Da}$ and its half-life is $\sim 2.5 \mathrm{~h}$ (26). The only skin troubles identified in the mouse model used in the present study were light reddish coloring and roughness of the skin. The skin is recognized as an attractive target for immunotherapy. Cutaneous administration has been associated with immunological benefits, including the induction of greater immune responses compared with those elicited by conventional routes of delivery $(21,22,27)$. Thus, IQM satisfies the requirements for transcutaneous administration.

In summary, combination therapy with transcutaneous IQM and oral sorafenib targeting VEGFR, PDGFR, flt3 and Ser/Thr kinases produced beneficial effects in an orthotopic mouse RCC model. IQM upregulated tumor-specific CTLs. Therefore, a combined therapy using IQM and TKIs may represent a promising strategy for patients with RCC, although this requires further investigation. Additional preclinical studies to determine optimal doses and schedules are also required.

\section{Acknowledgements}

The authors would like to thank Bayer AG (Leverkusen, Germany) and Mochida Pharmaceutical Co., Ltd. (Tokyo, Japan) for supplying the sorafenib and imiquimod, respectively. The present study was given proofreading support by FORTE Co., Ltd. (Tokyo, Japan), Japan's leading science communications company.

\section{References}

1. Topalian SL, Hodi FS, Brahmer JR, Gettinger SN, Smith DC, McDermott DF, Powderly JD, Carvajal RD, Sosman JA, Atkins MB, et al: Safety, activity, and immune correlates of anti-PD-1 antibody in cancer. N Engl J Med 366: 2443-2454, 2012.

2. Brahmer JR, Tykodi SS, Chow LQ, Hwu WJ, Topalian SL, Hwu P, Drake CG, Camacho LH, Kauh J, Odunsi K, et al: Safety and activity of anti-PD-L1 antibody in patients with advanced cancer. N Engl J Med 366: 2455-2465, 2012.

3. Royal RE, Levy C, Turner K, Mathur A, Hughes M, Kammula US, Sherry RM, Topalian SL, Yang JC, Lowy I and Rosenberg SA: Phase 2 trial of single agent Ipilimumab (anti-CTLA-4) for locally advanced or metastatic pancreatic adenocarcinoma. J Immunother 33: 828-833, 2010.

4. Hemmi H, Kaisho T, Takeuchi O, Sato S, Sanjo H, Hoshino K, Horiuchi T, Tomizawa H, Takeda K and Akira S: Small anti-viral compounds activate immune cells via the TLR7 MyD88-dependent signaling pathway. Nat Immunol 3: 196-200, 2002.

5. Peet NP, Baugh LE, Sunder S and Lewis JE: Synthesis and antiallergic activity of some quinolinones and imidazoquinolinones. J Med Chem 28: 298-302, 1985.

6. Lebwohl M, Dinehart S, Whiting D, Lee PK, Tawfik N, Jorizzo J, Lee JH and Fox TL: Imiquimod 5\% cream for the treatment of actinic keratosis: Results from two phase III, randomized, double-blind, parallel group, vehicle-controlled trials. J Am Acad Dermatol 50: 714-721, 2004.

7. Geisse JK, Rich P, Pandya A, Gross K, Andres K, Ginkel A and Owens M: Imiquimod 5\% cream for the treatment of superficial basal cell carcinoma: A double-blind, randomized, vehicle-controlled study. J Am Acad Dermatol 47: 390-398, 2002.

8. Naylor MF, Crowson N, Kuwahara R, Teague K, Garcia C, Mackinnis C, Haque R, Odom C, Jankey C and Cornelison RL: Treatment of lentigo maligna with topical imiquimod. Br J Dermatol 149 (Suppl 66): S66-S70, 2003.
9. Lyons JF, Wilhelm S, Hibner B and Bollag G: Discovery of a novel Raf kinase inhibitor. Endocr Relat Cancer 8: 219-225, 2001.

10. Karashima T, Komatsu T, Niimura M, Kawada C, Kamada M, Inoue K, Udaka K, Kuroda N and Shuin T: Novel combination therapy with imiquimod and sorafenib for renal cell carcinoma. Int J Urol 21: 702-706, 2014.

11. Vines $\mathrm{T}$ and Faunce T: Freedom of information applications as an 'evergreening' tactic: Secretary, department of health and ageing v iNOVA pharmaceuticals (Australia) Pty Ltd (2010) 191 FCR 573; [2010]FCA 1442. J Law Med 19: 43-52, 2011.

12. Karashima T, Inoue K, Fukata S, Iiyama T, Kurabayashi A, Kawada C and Shuin T: Blockade of the vascular endothelial growth factor-receptor 2 pathway inhibits the growth of human renal cell carcinoma, RBM1-IT4, in the kidney but not in the bone of nude mice. Int J Oncol 30: 937-945, 2007.

13. Visseren MJ, van Elsas A, van er Voort EI, Ressing ME, Kast WM, Schrier PI and Melief CJ: CTL specific for the tyrosinase autoantigen can be induced from healthy donor blood to lyse melanoma cells. J Immunol 154: 3991-3998, 1995.

14. Li Z, Oka Y, Tsuboi A, Fujiki F, Harada Y, Nakajima H, Masuda T, Fukuda Y, Kawakatsu M, Morimoto S, et al: Identification of a WT1 protein-derived peptide, WT1, as a HLA-A 0206-restricted, WT1-specific CTL epitope. Microbiol Immunol 52: 551-558, 2008.

15. Majewski S, Marczak M, Mlynarczyk B, Benninghoff B and Jablonska S: Imiquimod is a strong inhibitor of tumor cell-induced angiogenesis. Int J Dermatol 44: 14-19, 2005.

16. Vidal D, Matías-Guiu X and Alomar A: Efficacy of imiquimod for the expression of Bcl-2, Ki67, p53 and basal cell carcinoma apoptosis. Br J Dermatol 151: 656-662, 2004.

17. Kim CH, Ahn JH, Kang SU, Hwang HS, Lee MH, Pyun JH and Kang HY: Imiquimod induces apoptosis of human melanocytes. Arch Dermatol Res 302: 301-306, 2010.

18. Escudier B, Eisen T, Stadler WM, Szczylik C, Oudard S, Siebels M, Negrier S, Chevreau C, Solska E, Desai AA, et al: Sorafenib in advanced clear-cell renal-cell carcinoma. N Engl J Med 356: 125-134, 2007.

19. Santoni M, Berardi R, Amantini C, Burattini L, Santini D, Santoni $\mathrm{G}$ and Cascinu S: Role of natural and adaptive immunity in renal cell carcinoma response to VEGFR-TKIs and mTOR inhibitor. Int J Cancer 134: 2772-2777, 2014.

20. Cabrera R, Ararat M, Xu Y,Brusko T, Wasserfall C, Atkinson MA, Chang LJ, Liu C and Nelson DR: Immune modulation of effector $\mathrm{CD} 4+$ and regulatory $\mathrm{T}$ cell function by sorafenib in patients with hepatocellular carcinoma. Cancer Immunol Immunother 62: 737-746, 2013.

21. Delgado-Charro MB and Guy RH: Transdermal Drug Delivery. In: Hillary AM, Lloyd AW, Swarbrick J (eds). Drug Delivery and Targeting: For Pharmacists and Pharmaceutical Scientists. Taylor \& Francis, London and New York, pp189-214, 2001.

22. Mathias NR and Hussain MA: Non-invasive systemic drug delivery: Developability considerations for alternate routes of administration. J Pharm Sci 99: 1-20, 2010.

23. Oertel W, Ross JS, Eggert K and Adler G: Rationale for transdermal drug administration in Alzheimer disease. Neurology 69 (4 Suppl 1): S4-S9, 2007.

24. Seeberger LC and Hauser RA: Optimizing bioavailability in the treatment of Parkinson's disease. Neuropharmacol 53: 791-800, 2007.

25. Santini B, Zanoni I, Marzi R, Cigni C, Bedoni M, Gramatica F, Palugan L, Corsi F, Granucci F and Colombo M: Cream formulation impact on topical administration of engineered colloidal nanoparticles. PLoS One 10: e0126366, 2015.

26. Soria I, Myhre P, Horton V, Ellefson P, McCarville S, Schmitt K and Owens M: Effect of food on the pharmacokinetics and bioavailability of oral imiquimod relative to a subcutaneous dose. Int J Clin Pharmacol Ther 38: 476-481, 2000.

27. Stein P, Gogoll K, Tenzer S, Schild H, Stevanovic S, Langguth P and Radsak MP: Efficacy of imiquimod-based transcutaneous immunization using a nano-dispersed emulsion gel formulation. PLoS One 9: e102664, 2014. 